
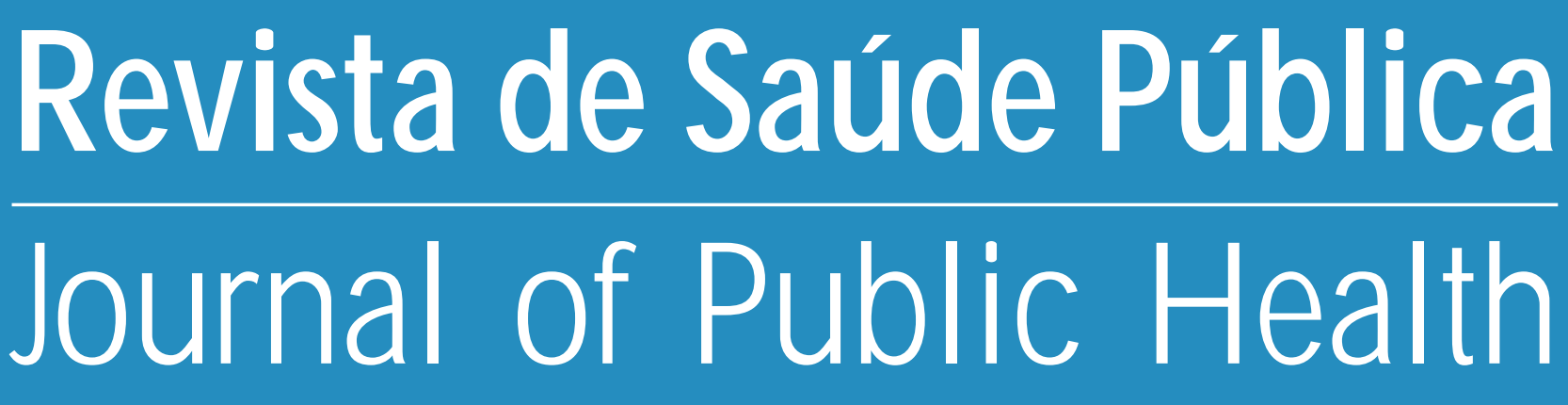

\title{
Tolerancias y resistencias: el aborto desde la perspectiva de las parteras tradicionales de un área rural de México
}

\section{Tolerance and resistance: abortion from the point of view of traditional midwives in a rural area of Mexico}

\section{Julia Blanco-Muñoz y Xochitl Castañeda-Camey}

Departamento de Transición Epidemiológica del Centro de Investigación en Salud Pública del Instituto Nacional de Salud Pública (IN SP). Cuernavaca, Morelos, México (JBM), Departamento de Salud Sexual y Reproductiva del Centro de Investigación en Salud Pública del IN SP. Cuernavaca, Morelos, M éxico (XCC) 


\title{
Tolerancias y resistencias: el aborto desde la perspectiva de las parteras tradicionales de un área rural de México*
}

\section{Tolerance and resistance: abortion from the point of view of traditional midwives in a rural area of Mexico}

\author{
Julia Blanco-M uñoz y Xochitl Castañeda-Camey
}

Departamento de Transición Epidemiológica del Centro de Investigación en Salud Pública del Instituto Nacional de Salud Pública (INSP). Cuernavaca, Morelos, M éxico (JBM), Departamento de Salud Sexual y Reproductiva del Centro de Investigación en Salud Pública del INSP. Cuernavaca, Morelos, México (XCC)

\section{D escriptores}

Aborto inducido.

Conocimientos, actitudes y práctica. Parteras tradicionales.

\section{Resumen}

\section{Objetivo}

Conocer la percepción, recursos y prácticas que sobre el aborto tienen las parteras de un área rural mexicana, el Municipio de Yecapixtla, Morelos, situado en la parte central de México.

\section{Métodos}

Se utilizó método cualitativo, realizándose entrevistas en profundidad, grupos focales y observación participativa. Los tópicos indagados fueron aspectos socioculturales, sexualidad, reproducción y aspectos de salud en relación al aborto. Se entrevistó en profundidad a 9 parteras y se realizó un grupo focal en el que participaron 16 parteras.

\section{Resultados}

Éstos dan cuenta de una profunda actitud de rechazo ante el aborto, inducido o espontáneo. El primero se conceptúa como un pecado grave y el segundo como un fracaso en la principal función de la mujer en estas comunidades, la reproducción. Las mujeres que abortan son llamadas "puercas", "cochinas" o "perras" y las parteras se muestran reacias a atenderlas. Sin embargo, es común entre las mujeres de la comunidad la práctica de la "regulación de la regla", es decir, la utilización de sustancias que hacen que aparezca la menstruación si ésta se ha retrasado. Dicha práctica no se considera abortiva.
Correspondencia para/Correspondence to:

Julia Blanco-Muñoz

Avda. Universidad 655

Col. Sta. María Ahuacatitlán, CP:62508

Cuernavaca, Morelos, México.

E.mail: jblanco@insp3.insp.mx
* Subvencionado pelo IPAS, México.

Recibido en 6.4.1998. Reapresentado en 17.11.1998. Aprobado en 1.12.1998. 


\section{Keywords}

Abortion, induced.

Knowledge, attitudes, practice. Midwives, practical.

\section{Descritores}

Aborto induzido.

Conhecimentos, atitudes e prática. Parteira leiga.

\section{Conclusiones}

El conocimiento del mundo de significaciones populares en torno al aborto es imprescindible para construir modelos y estrategias efectivas que, desde los servicios institucionales de salud, refuercen los vínculos entre éstos y las parteras tradicionales, de manera que se mejore el acceso a servicios y la calidad de la atención a las mujeres.

\section{Abstract}

\section{Objective}

An evaluation of the perception, resources and practices regarding abortion of traditional midwives in a rural area in the municipality of Yecapixtla, state of Morelos, located in the central region of Mexico.

\section{Methods}

A qualitative methodology consisting of a detailed interview, focal groups and participating observation, was used. The subjects investigated were socio-cultural aspects, reproduction, sexuality and health related to abortion. Nine midwives were interviewed and a focal group was formed in which 16 midwives participated.

Results

The results demonstrated a profound rejection of abortion whether inducted or spontaneous. The former was considered a major sin and the latter a serious failure of a woman's reproductive function. Women who abort are called "pigs", "hogs" or "bitches" and the midwives are reluctant to attend them. However, a common practice among the women in the community is to "regulate the menstruation", that is, to use substances that provoke menstruation when this is delayed. This specific practice is not considered abortive by these women.

\section{Conclusion}

Local popular beliefs about abortion are indispensible for the construction of effective strategies, which when provided by the institutional health services, reinforce the bonds between these and the traditional midwives in such a way as to increase accessibility to the health services as well as the quality of care to women.

\section{Resumo}

\section{Objetivo}

Conhecer a percepção, recursos e práticas que as parteiras tradicionais, de uma área rural mexicana, possuem sobre o aborto.

\section{Métodos}

Usou-se o método qualitativo com entrevistas em profundidade, grupos focais e observação participativa. As questões versaram sobre aspectos socioculturais, sexualidade, reprodução e aspectos de saúde relacionados ao aborto. Entrevistaramse em profundidade 9 parteiras e formou-se um grupo focal com a participação de 16 parteiras.

\section{Resultados}

Encontrou-se uma atitude de não aceitação do aborto, induzido ou espontâneo. As mulheres que abortam são chamadas de "porcas", "imundas" ou "cadelas" e as parteiras mostram-se titubeantes em atendê-las. No entanto, é comum a prática da "regulação da menstruação" entre as mulheres da comunidade, ou seja, utilizam substâncias que induzem a menstruação quando a mesma está atrasada.

\section{Conclusões}

O conhecimento de significados populares em torno do aborto é imprescindível para a construção de estratégias que reforcem os vínculos entre serviços institucionais de saúde e as parteiras tradicionais e para melhorar a qualidade da assistência às mulheres. 


\section{INTRODUCCIÓN}

En todo el mundo, el nacimiento de un niño es un hecho que se festeja. Sin embargo, miles de mujeres viven el proceso del embarazo no como el acontecimiento gozoso que debería ser sino como una experiencia dolorosa que, incluso, puede terminar en la muerte. En casi todas las sociedades, la celebración de la vida es el tema dominante, mientras que los aspectos sombríos de la procreación, se envuelven en un manto de silencio y sólo son conocidos por las mujeres y por quienes las atienden ${ }^{13}$. Uno de estos aspectos es el aborto, sin cuya referencia, cualquier visión panorámica de los temas sobre salud sexual y reproductiva resultaría incompleta ${ }^{1}$.

En América Latina se producen 4 millones de abortos anuales ${ }^{6}$. En México, según datos de la Encuesta Nacional sobre Fecundidad y Salud ${ }^{4}$, una de cada seis mujeres en edad fértil declaró haber tenido un aborto espontáneo o inducido alguna vez en su vida.

Aunque es difícil evaluar la contribución del aborto a la mortalidad materna, se estima que, en América Latina, las complicaciones del aborto ilegal son la primera causa de muerte en mujeres entre 15 y 19 años ${ }^{8}$; en México, para 1995, el aborto ocupó el cuarto lugar como causa de muerte materna ${ }^{14}$.

Aparte de la magnitud del problema en términos numéricos, el aborto marca la experiencia reproductiva posterior de las mujeres. En el caso del aborto espontáneo, generalmente, su etiología no llega a descubrirse, lo que ocasiona en la mujer una profunda ansiedad en futuros embarazos y un manejo inadecuado de los mismos ${ }^{16}$.

La situación del aborto inducido es aún mas compleja; puesto que en muchos países, entre ellos México, existe una legislación restrictiva y punitiva al respecto, suelen realizarse de forma clandestina y, muchas veces, bajo condiciones deplorables desde el punto de vista sanitario y psicológico, lo que incrementa el riesgo de complicación y de muerte ${ }^{13}$. Este riesgo no se distribuye uniformemente entre la población, siendo más alto en mujeres pobres y residentes en áreas rurales de países en desarrollo, quienes tienen menos posibilidad de acceder a servicios de salud que les presten atención adecuada y oportuna. Por ello, para atenuar la escasez de servicios en dichas áreas y aprovechar los recursos existentes en ellas, se ha intentado integrar a los terapeútas tradicionales en el sistema de salud; dentro de ellos, la partera empírica es una figura fundamental en la atención del embarazo, parto y puerperio ${ }^{2}$; en México, se han desarrollado cursos de capacitación para parteras con el fin de disponer de un recurso humano adiestrado accesible geográfica, económica y culturalmente a aquellas mujeres. Lo último es especialmente importante ya que la concepción, el aborto y el ciclo reproductivo no son fenómenos biológicos aislados y los aspectos culturales(mitos y creencias) y espirituales ligados a ellos ejercen gran influencia sobre el resto de actividades e instituciones sociales, por lo que deben ser tomados en cuenta al organizar los servicios de salud.

Estudios previos han documentado la labor de las parteras tradicionales en la atención del embarazo, parto y puerperio, sin embargo se sabe poco del papel que juegan en otros eventos de la salud sexual y reproductiva, como es el caso del aborto. Por ello, el objetivo de este trabajo fue investigar los conceptos, recursos y prácticas que sobre este problema tienen las parteras de una comunidad rural del estado de Morelos(México), estado donde el aborto supone la cuarta causa de muerte materna y representa una proporción importante de la morbilidad hospitalaria ${ }^{7}$.

El presente estudio forma parte de un proyecto más amplio*, cuya finalidad es mejorar el acceso a servicios de salud y la calidad de la atención a mujeres con aborto, mediante la implementación de una estrategia de capacitación a parteras rurales.

\section{MÉTODOS}

El estudio se realizó en Yecapixtla (Morelos), donde todavía numerosas mujeres son atendidas por parteras tradicionales.

Se empleó metodología cualitativa; mediante enfoque constructivista se exploraron las significaciones que dan las parteras tradicionales al aborto y los aspectos socioculturales donde se dimensionan de forma profunda y contextualizada.

Los instrumentos utilizados fueron: entrevistas en profundidad y grupos de discusión. También se realizaron observaciones participativas durante 6 meses de trabajo

* Esse proyecto fue diseñado por el Instituto Nacional de Salud Pública en colaboración con la Subsecretaría de Salud del Estado de Morelos. 
de campo (Noviembre 1995-Abril 1996). Estas herramientas dieron acceso a los lenguajes (oral, corporal, coloquial, metafórico e incluso el silencio) usados en relación al ciclo reproductivo y al aborto.

El análisis fue interpretativo. La principal unidad de análisis fueron las conversaciones con las parteras. Los determinantes sociales sobre la percepción del aborto se analizaron a partir de dos categorías: la personal y la cultural. Los determinantes personales incluyen el nivel socio-económico, los paradigmas populares sobre roles de género, los aspectos emocionales, las creencias sobre salud, los patrones de búsqueda de atención y las motivaciones a las que se alude para practicar un aborto inducido. En cuanto a los determinantes culturales se enfatizó sobre los mitos, tabúes y leyendas, el peso de la religión, la percepción del cuerpo, la normatividad respecto al deber ser y la doble moral.

Selección de Comunidades: se eligió un municipio del estado de Morelos. Los criterios de selección fueron los siguientes:

- municipio con comunidades campesinas.

- cuya población fuera menor de 2.500 habitantes.

- que tuviera acceso a no más de 1 hora de la capital del estado.

- que estuviera a máximo 1,5 horas de un hospital de segundo nivel.

- que hubiera parteras tradicionales capacitadas.

- con índice de marginación municipal bajo3.

- que contara con medios de transporte colectivos, para poder tener acceso en forma fluída al trabajo de campo.

Selección de Informantes Claves: se trabajó con parteras tradicionales que hubieran recibido cursos de capacitación sobre salud sexual y reproductiva. Se les presentó el proyecto y se garantizó el anonimato de sus testimonios. Se pudo entrevistar en profundidad a nueve parteras y se formó, además, un grupo de discusión con 16 parteras.

\section{Técnicas de Investigación}

a) Grupo de discusión. Fue manejado por una antropóloga. Se trabajó con temas de motivación grupal en la comprensión y valoración de conceptos clave para captar las apreciaciones colectivas en torno al aborto. Tuvo una duración de 2,5 horas. La discusión fue grabada y posteriormente transcrita.

b) Entrevista en profundidad. Se utilizó una guía de entrevista con preguntas abiertas. La entrevista se realizó en varias sesiones, con una duración aproximada de 6 horas en total por cada partera. Para su conducción se manejaron manuales de metodologías cualitativas ${ }^{5}$.

El vaciado de datos se efectuó mediante una matríz temática construída a lo largo del estudio, ello permitió analizar los aspectos comunes y las divergencias más significativas.

\section{RESULTADOS}

\section{Descripción de la Comunidad}

Yecapixtla (lugar donde sopla el viento delgado) pertenece al estado de Morelos, en la región central de México. Previo a la conquista española, poblaron el municipio grupos venidos de Xochimilco, tlahuicas y mexicas, sucesivamente. Durante el dominio español arrivaron órdenes religiosas (agustinos). La región fue escenario de luchas durante la revolución mexicana. Actualmente, la principal actividad económica es la agricultura, desarrollada principalmente por los hombres; las mujeres con actividad económica se dedican a servicios comunales y al comercio. El municipio cuenta con siete centros de salud, coordinados por una Jurisdicción Sanitaria. Además, existe un número indefinido de terapeutas tradicionales que proporcionan atención a sus habitantes, especialmente en las localidades más aisladas, cuyas mujeres suelen acudir con las parteras para atenderse durante el embarazo y el parto.

\section{Características G enerales de las Informantes}

La edad promedio de las parteras fue de 46 años. Dos de las parteras eran solteras y el resto estaban o habían estado casadas o en unión libre; dos no tenían hijos. Todas ellas habían recibido algún curso de capacitación en atención de embarazo, parto y puerperio por parte de instituciones del sector salud. El promedio mensual de embarazadas que atienden es de cinco, con un rango que va desde dos hasta quince.

\section{Conceptualización del Aborto Espontaneo e Inducido}

Todas las informantes distinguen entre aborto espontáneo y aborto inducido. El primero lo conceptualizan como un fracaso de la mujer en su función de género principal: la reproductora. Algunas parteras afirman que las mujeres que abortan espontáneamente "no saben tener hijos" o "no se cuidan y por eso abortan" y culpan a la mujer por la pérdida.

En cuanto al aborto inducido, dado el orden moral y/o normativo que impera en estas comunidades, las entrevistadas lo consideran un asesinato o un pecado grave. Los apelativos con los que la comunidad se refiere a las mujeres que abortan voluntariamente son "perras", "cochinas" o "puercas" y al hecho de abortar lo denominan: "comerse el hijo" o "tirar el 
hijo". Cuando una mujer aborta repetídamente, aunque se trate de abortos espontáneos, también se hace acreedora de estos apelativos "porque la gente sospecha que debe estar haciendo algo para tirar el hijo".

\section{Causas del Aborto Espontáneo}

Las parteras atribuyen el aborto espontáneo a diversas causas; una de las más mencionadas son los llamados "antojos", deseos compulsivos que la embarazada siente generalmente (aunque no siempre) por algún alimento. Cabe señalar que en otros lugares, como en América y Europa, la denominación se extiende, por metonimia, al signo externo que se supone aparece en el niño como consecuencia de un antojo no satisfecho, casi siempre una mancha corporal; se cree que es el niño y no la madre quien desea satisfacer el antojo ${ }^{10}$. De no satisfacerlo se puede producir aborto espontáneo. El remedio es la satisfacción del antojo o, en su defecto, la sustitución de lo deseado por otro alimento, por ejemplo las tortillas de maíz con sal.

Otras causas a las que atribuyen el aborto espontáneo son la movilidad de ciertas partes del cuerpo: cadera o cintura "abiertas" y la "caída de la matríz o de los ovarios"*. Para curar estas afecciones, las parteras disponen de recursos que combinan remedios herbolarios con terapias mecánicas (sobada, manteada, masaje, colgar a la mujer por los pies) y baños de temazcal. Generalmente, las curaciones se acompañan de rituales de eficacia simbólica y/o mágica.

El trabajo excesivo y pesado que realizan las mujeres durante el embarazo y el maltrato de la mujer por su pareja masculina se mencionaron también como causas de aborto espontáneo.

\section{Motivos del A borto Inducido}

Desde el punto de vista de estas parteras, los motivos que inducen a una mujer a abortar intencionalmente, varían dependiendo de si es soltera o si está casada o con pareja estable. En el primer caso, el motivo fundamental es el temor ante la reación familiar y social, que suele ser de rechazo ante un embarazo fuera de una unión estable. Muchas veces, "el novio se desentiende del problema y al encontrarse sola, sin recursos y con un hijo, dijeron, las mujeres deciden abortar"; "también sucede que es el novio el que obliga a la mujer a abortar", o "ella sabe que él no quiere hijos y decide abortar porque piensa que, si no, la va a abandonar". Es frecuente que el hombre no sepa que su pareja está embarazada. Las mujeres también piensan que si tienen un hijo solteras, es difícil encontrar luego una pareja formal, lo que, según las parteras, puede influir en la decisión de abortar.

En el caso de las mujeres casadas, los motivos para buscar un aborto inducido son las desavenencias conyugales y los problemas económicos. No obstante, las entrevistadas opinan que estos motivos no justifican el aborto: "si no se llevan bien con el marido, que lo dejen, pero que no aborten", "mucha gente desea tener un hijo, si no lo quieren, que lo regalen", "si una casada quiere abortar debe ser porque el niño no es del marido", "problemas económicos siempre los ha habido", son sus testimonios.

\section{Circunstancias que Justifican el Aborto Inducido}

Dos parteras mencionaron que existen circunstancias (violación, malformación en el feto, riesgo para la vida de la madre) que justificarían la realización de un aborto, el resto manifestó que ni aún así sería justificable. Por ejemplo, en el caso de violación afirman que el bebé no es responsable de la misma y que su vida debe respetarse. En caso de malformaciones congénitas, aducen que "ellas no pueden saber si el niño está mal antes de nacer”, por lo cual no se debe hacer un aborto.

En consonancia con lo anterior, cuando se les preguntó su opinión acerca de la liberalización del aborto todas se manifestaron en contra de la misma porque "los abortos inducidos se multiplicarían pues sería muy fácil para las mujeres hacérselos".

\section{Actitud de las Parteras ante las Mujeres que Acuden a Ellas para Solicitar un Aborto}

Las parteras reconocen que, con cierta frecuencia, acuden a ellas mujeres solicitando un aborto, si bien todas ellas manifestaron que no practican abortos y la mayoría dice desconocer maniobras o remedios abortivos. Dos parteras dijeron que ellas sí co-

* Dentro de la percepción del cuerpo que impera en estas zonas rurales de Morelos, cabe señalar la movilidad que se le atribuye a los órganos: la fontanela se cae, los pulsos cambian de lugar, los órganos se suben o bajan o se abren... Esto tiene fuerte correlación con la cosmovisión prehispánica. 
nocían "lo bueno" para abortar, pero que no lo proporcionaban a las mujeres; por el contrario, "cuando alguna viene con esa petición le damos remedios para que se le pegue más"(es decir, para que no aborten).

Las actitudes que asumen cuando llegan mujeres con esta demanda van desde el franco rechazo hasta el consejo a la mujer, sugiriéndole que medite su decisión y que tenga su hijo; para disuadir a estas mujeres apelan, también, a los riesgos que implica un aborto.

Las parteras manifiestan que las mujeres que desean abortar suelen acudir solas o con alguna otra mujer, siendo excepcional que la pareja masculina las acompañe.

Cuando se les preguntó si, dado que ellas no atendían las demandas de estas mujeres, sabían dónde se dirigían para realizarse el aborto, dijeron que lo desconocían.

\section{Recursos Abortivos U tilizados en la Comunidad}

Pese a que las parteras dijeron desconocer recursos abortivos, cuando se les preguntó acerca de los utilizados por las mujeres en la comunidad fueron más explícitas y mencionaron:

- Zoapatle (montanoa tomentosa), el cual se usa también para acelerar el parto. El zoapatle es más eficaz si se complementa con chocolate muy caliente y/o con un baño muy caliente.

- Las inyecciones de hormonas (Lutoginestril F-5).

- La sonda.

- "la raspa"(legrado).

- La "succión con una jeringa" (aspiración manual endouterina)

- Cargar cosas pesadas o dejarse caer de lugares elevados.

Sobre recursos abortivos populares en las Américas existen diversos trabajos*.

\section{Complicaciones del Aborto}

Las complicaciones del aborto que citaron fueron la hemorragia y la infección, a corto plazo. Como complicación a largo plazo, se mencionó reiteradamente la esterilidad, de manera que sobre la mujer esteril recae la sospecha de que alguna vez se hizo un aborto. Cabe mencionar que sobre la mujer esteril pesa un fuerte estigma y son nombradas con diversos epitetos: "mulas", "jorras" y "machorras", de carácter profundamente peyorativo.

Cuando las parteras reciben mujeres que presentan alguna complicación por aborto, sea espontáneo o provocado, generalmente las remiten a algún centro médico sin realizar ninguna maniobra. Sólo una de las parteras mencionó que ponía suero glucosalino en tanto la mujer podía recibir atención especializada. Las parteras dicen actúar de esta forma por desconocimiento de la terapia adecuada en estas situaciones, porque en los cursos de capacitación se les ha dicho que es la conducta que deben observar y por último, y muy importante, por el temor a que se piense que son "aborteras", ya que la condena social y moral que late en torno al aborto en estas comunidades es muy fuerte. Además, todas saben que en México el aborto inducido, salvo excepciones, es ilegal.

Paradójicamente, todas las parteras mostraron interés en aprender las terapias que permitan que mujeres con complicaciones postaborto lleguen en las mejores condiciones a los centros donde puedan ser atendidas de forma adecuada.

\section{Aborto y Religión}

La influencia de la religión católica se percibe en las opiniones expresadas sobre el aborto inducido. Como se mencionó, éste se concibe como un pecado grave y la mujer que aborta intencionalmente o aquellos que la ayudan a abortar "se echan a la lumbre"(en referencia a las llamas del infierno). Afirman que Dios va a pedir cuentas por los hijos perdidos, lo cual nos remite a leyendas populares mexicanas como la de la "Llorona", personaje que abandonó a sus hijos a la orilla del río y a la que Dios preguntó por ellos, y ella, al no saber donde estaban, fue condenada a vagar eternamente hasta encontrarlos.

\section{La Práctica de la Regulación de la Regla}

Pese a que todas las entrevistadas condenan rotundamente el aborto, existe una práctica frecuentemente utilizada por las parteras y las mujeres de la comunidad que es la llamada "regulación de la regla"; consiste en utilizar sustancias (generalmente herbola-

\footnotetext{
* Se puede consultar el trabajo de MP David y S Pick de Weis, titulado "El aborto en las Américas", publicado en "Salud Reproductiva en las Américas", OPS, Washington, DC, 1992, editado por López G y otros.
} 
rias) para que baje la menstruación cuando se ha retrasado; se piensa que la sangre retenida es nociva para el organismo y que la regulación sirve para limpiarlo. Dicha práctica no es considerada abortiva.

Las parteras la recomiendan cuando el retraso es de menos de un mes y la mujer no sabe si está o no embarazada. Se piensa que si la regla se presenta es que no había un embarazo. Por el contrario, si no aparece la menstruación, la mujer está embarazada y tiene que resignarse "porque Dios quiere que ese niño nazca". Desde este punto de vista, la regulación menstrual sería una forma de diagnosticar la gestación.

Las parteras disponen de un amplio catálago de sustancias destinadas a regular la regla:

- El té de canela con orégano.

- El zoapatle con chocolate caliente, o con epazote o zábila.

- El té de yerbadulce y el de manzanilla.

- Inyecciones de hormonas.

- El ácido acetil salicílico con jugo de limón.

\section{Ideas sobre la G estación y el Desarrollo del Feto}

Las parteras conciben la formación del nuevo ser como un proceso en el que el carácter humano, propiamente dicho, se adquiere cuando se forman los huesos, lo que desde su percepción sucede a los tres o cuatro meses de gestación. Entonces, dicen, el bebé es como una "ranita" y están formados sus órganos. Esto se refiere a los fetos masculinos porque se piensa que las niñas son como una bola de sangre, sin huesos, hasta por los menos los seis meses de embarazo. Lo anterior no influye en el hecho de condenar el aborto en cualquier etapa del embarazo (independientemente del sexo).

\section{Sobre Planificación Familiar}

Dado que se trabajó con parteras adiestradas, todas conocen a grandes rasgos los principales métodos de planificación familiar(PF), materia que forma parte importante de los cursos de capacitación. Sin embargo, la mayoría se muestran reacias a la utilización de los mismos.

Las parteras piensan que los anovulatorios hacen daño a las mujeres, que les puede dar cancer o que se ponen "feas y flacas". Del dispositivo intrauterino (DIU) opinan que puede lastimar a la mujer y que pocas lo aguantan. En cuanto a los métodos de barrera (se mencionó el preservativo) manifiestan que los hombres casi nunca quieren utilizarlos.

Según las parteras, a los hombres de estas comunidades no les gusta que sus mujeres "se planifiquen". Dicen que, muchas veces, aquellas que han aconsejado métodos anticonceptivos a sus pacientes han recibido amenazas de las parejas de las mujeres.

Las parteras afirman, por su parte, que las parejas se unen para tener los hijos que Dios les mande y que si las mujeres no quieren quedar embarazadas es mejor que no tengan relaciones sexuales.

En el caso de que alguna mujer les pida consejo sobre PF la envían al centro de salud para que allí se informe.

\section{DISCUSIÓN}

En primer lugar, hay que señalar que el carácter cualitativo del estudio no permite hacer generalizaciones ni inferencias numéricas, puesto que su objetivo no es la representatividad estadística.

De las entrevistas resalta, en primer término, la marcada actitud de rechazo hacia el aborto, especialmente cuando se trata de aborto inducido, ya que representa una oposición contra el orden moral y social. Se traslucen, además, varias ideas sobre las cuales gira el discurso: el principal objetivo de la relación de pareja es la reprodución; otros aspectos de la relación, tales como el placer o las demostraciones del afecto son muy tabuados y poco exteriorizados, al menos a nivel del discurso oral. Asímismo, la normativa social se encamina a presionar dentro del "deber ser", la función reproductiva de la pareja, con énfasis en la mujer. De ahí que la concepción sea uno de los actos fundamentales que justifican (y a partir de los cuales se estructuran) los roles de género y permiten la continuidad del grupo social y doméstico. En este contexto, tanto para el hombre como para la mujer es deseable tener un número elevado de hijos. Para el primero, éstos representan la forma de manifestar su virilidad ante el grupo social. Para la mujer, en un ámbito dónde prácticamente su único proyecto de vida es la formación de una familia, el matrimonio y la fertilidad son la principal forma de adquirir prestigio y respeto; por ello, el aborto es un tema tabuado, pues junto con la esterilidad expresa el fracaso en la principal función que se atribuye a la pareja, y en particular a la mujer. 
En el caso de las parteras hay que considerar, además, que desde hace siglos han sido las encargadas de ayudar a las mujeres a traer sus hijos al mundo y que son muy respetadas en sus comunidades, donde los valores tradicionales y culturales permanecen fuertemente arraigados; ello explica su rechazo ante el aborto y su resistencia a proporcionar información sobre planificación familiar. Tampoco hay que olvidar que la mayoría son de edad relativamente avanzada, por lo que no es sencillo modificar sus creencias y prácticas.

Por otra parte, tomando en cuenta la penalización legal, social y moral que pesa sobre el aborto, no debe sorprender el hecho de que las parteras se abstengan de atender a mujeres que acuden a ellas con una complicación postaborto. En este sentido, si se desea que las parteras se involucren en la atención de estas mujeres, es necesario que desde las instancias oficiales se les apoye y garantice que no tendrán problemas de tipo jurídico.

Como se observó, pese a la oposición al aborto inducido, es común y aceptada culturalmente la práctica de la regulación menstrual. Obviamente, existe un traslape entre lo que es aborto y regulación de la regla; sin embargo, en estas comunidades se les atribuye un significado diferente, de manera que dicha práctica al no ser nombrada ni interiorizada como abortiva, no conlleva las cargas morales y sociales de lo que se cataloga como un delito: la interrupción de un embarazo. Otras cultu-

\section{REFERENCIAS}

1. Antrobus ACK. Temas contemporáneos en la atención de la salud maternoinfantil. Bol Oficina Sanit Panam 1986; 100:387-96.

2. Castañeda X, Garcia C, Romero X, Nuñez RM, Gonzalez D, Langer A. Traditional birth attendants in México: advantages and inadequacies of care for normal deliveries. Soc Sci Med 1986; 43:199-207.

3. CONAPO. Indicadores socioeconómicos e indice de marginación, 1990. México (DF): 1993. p. 203.

4. Encuesta Nacional sobre Fecundidad y Salud, 1987. Dirección General de Planificación Familiar, Secretaría de Salud, México, 1988.

5. Fontana A, Frey J. Interviewing: the art of science. In: Handbook of qualitative research. London: Sage Publications; 1994. p. 361-4.

6. Henshaw SK. Induced abortion: a world review. Int Fam Plann Perspect 1990; 22:76-89.

7. INEGI. Gobierno del Estado de Morelos. Anuario estadístico del estado de Morelos. Morelos; 1996. p. 171-5.

8. Jacobson JL The global politics of abortion. Washington (DC): World-watch Institute; 1990. (Worldwatch Paper, 97). ras también hacen uso de la citada práctica con propósitos bastante similares ${ }^{11,15}$.

Para concluir, abortar no es un hecho aislado, sino que se inserta dentro de una explosión de significados e imágenes que marcan la apropiación de la experiencia ${ }^{12}$; a partir del conocimiento de este mundo de significados en torno al aborto se podrán construir estrategias efectivas desde los servicios institucionales de salud. Si éstas son apropiados y aceptables para los destinatarios, se evitarán conflictos innecesarios con los patrones culturales ${ }^{9}$. Asimismo, es importante reforzar los vínculos entre dichos servicios y las parteras, de manera que éstas no se limiten a negarse a atender a mujeres que acuden con una complicación postaborto, sino que dentro de un sistema organizado de referencia y contrarreferencia, estén capacitadas para derivar a las mujeres en las mejores condiciones posibles y a hacer un seguimiento y control de ellas una vez que regresan a la comunidad.

\section{AGRADECIMIENTOS}

A las siguientes personas por su cooperación en el trabajo de campo: Nicté Castañeda, Dolores González; ana Rosa Maldonado y Laura Rodríguez; a Debborah Billings, Virginia Chambers, Xochitl Romero y Andrea Saldaña por su ayuda en el análisis preliminar de los resultados y en la revisión del documento; y especialmente a todas las parteras por su colaboración y sus conocimientos.

9. Kelly I. El adiestramiento de parteras en México, desde el punto de vista antropológico. América Indígena 1955; 25:109-17.

10. Mellado V, Zolla C, Castañeda X. La atención al embarazo y el parto en el medio rural mexicano. México (DF): Centro Interamericano de Estudios de Seguridad Social; 1989. p. 99-102.

11. Renne EP. The pregnancy that doesn't stay: the practice and perception of abortion by Ekiti Yoruba women. Soc Sci Med 1996; 42:483-94.

12. Rivas M, Amuchastegui A. Voces e historias sobre el aborto. Mexico (DF): The Population Council; 1996.

13. Royston E, Armstrong S. Prevención de las mortalidad materna. Ginebra: Organización Mundial de la Salud 1991.

14. Secretaria de Salud. Dirección General de Estadística e Informatica. Mortalidad 1995. México, DF; 1996. p:147-8.

15. Sobo EJ. Abortion traditions in rural Jamaica. Soc Sci Med 1996; 42:495-508.

16. Wolf G, Horger E. Indications for examination of spontaneous abortion specimens: a reassessment. Am J Obstet Gynecol $1995 ; 173: 1364-8$ 